\title{
КЛИНИЧЕСКАЯ ЗНАЧИМОСТЬ НЕИНФОРМАТИВНОГО И НЕОПРЕДЕЛЕННОГО ЗАКЛЮЧЕНИЙ ЦИТОЛОГИЧЕСКОГО ИССЛЕДОВАНИЯ УЗЛА ЩИТОВИДНОЙ ЖЕЛЕЗЫ
}

\author{
' Яновская Е.А., ' Яновская М.Е., ${ }^{2}$ Александрова С.В., ${ }^{3}$ Александров Ю.К. \\ 'ГБУЗ ЯО ОКБ, Ярославль \\ 2ГУ3 «Детская поликлиника №5», Ярославль \\ ${ }^{3}$ ФГБОУ ВО ЯГМУ Минздрава России, Ярославль
}

Цитологическое исследование является одним из основных при определении лечебной тактики при узловом зобе. Система The Bethesda System for Reporting Thyroid Cytopathology (Bethesda) четко регламентирует действия врачей в зависимости от выявленной цитологической картины. Однако имеются категории Bethesda, имеющие неоднозначную трактовку.

ЦЕЛЬ: конкретизировать клиническое значение категорий Bethesda 1 и Bethesda 3 цитологического исследования щитовидной железы с позиции прогнозирования лечебной тактики.

МАТЕРИАЛЫ И МЕТОДЫ: изУчены данные цитологического исследования у 1606 человек с узловыми образованиями щитовидной железы. Всем пациентам были выполнены ультразвуковое исследование с градацией по системе TIRADS и цитологическое исследование с градацией по системе Bethesda. B последующем все пациенты были оперированы по различным показаниям. Данные цитологического и УзИ сравнивались с результатами послеоперационного патоморфологического исследования.

PЕЗУЛЬТАТЫ: при анализе результатов предоперационного обследования у 110 человек (6,8\%) были получены заключение Bethesda1 (79 человек (4,9\%)) и Bethesda3 (31 человек (1,9\%)). При общей тенденции отечественных и зарубежных рекомендаций к динамическому наблюдению и повторным ТАПБ у этих категорий пациентов по данным послеоперационного патоморфологического исследования у 15 человек (13,6\%) был выявлен рак щитовидной железы, а у 17 (15,5\%) - аденома щитовидной железы. Учитывая значительное число опухолевых поражений щитовидной железы с указанными категориями Bethesda, были проанализированы данные других методов исследований, в частности УзИ. Было установлено, что у 16 больных по данным УЗИ был TIRADS 2 (у 5 - рак щитовидной железы, у 11 - аденома щитовидной железы), а у 16 - TIRADS4 (у 10 - рак щитовидной железы, а у 6 - аденома щитовидной железы). У пациентов с TIRADS3 и TIRADS5-6 неинформативные и неопределенные заключения цитологического исследования получены не были. С учетом того, что в группе больных с TIRADS 2 преобладало заключение Bethesda 1 (неинформативный мазок), а в группе TIRADS4 - заключение Bethesda3 (атипия неопределенного значения), необходимо признать, что пациенты с сочетанием TIRADS4 и Bethesda3 имеют наибольшую вероятность злокачественного поражения щитовидной железы (7,9\%). Таким образом, эта группа пациентов при стратификации рисков является наиболее угрожаемой по наличию рака ЩЖ и должна рассматриваться, как кандидат на хирургическое лечение. В группе с TIRADS2 общая частота выявления рака щитовидной железы составила 4,9\% (38 человек), в этой группе лишь у 5 человек $(0,6 \%)$ имелись градации Bethesda1 и Bethesda3. При статистическом сопоставлении данных цитологического и ультразвукового исследования (ROC- анализ) была подтверждено мнение, что решение о лечебной тактике должно строиться на комплексной оценке результатов исследований.

ВЫВоды: при получении заключения категорий Bethesda 1 и Bethesda 3 решение о лечебной тактике должно основываться на комплексной оценке данных обследования. Наиболее вероятной в плане онкологического риска является категория пациентов TIRADS4- Bethesda3. Этим пациентам необходимо рекомендовать оперативное лечение. Наименее вероятной по наличию рака щитовидной железы является группа пациентов TIRADS2- Bethesda 1, у них возможно наблюдение и проведение повторной ТАПБ. 